\title{
Vortex Pinning in Ferromagnet/Superconductor Bilayers - the Dependence on the Ferromagnetic Layer and Temperature
}

\author{
Z. Adamus, M.Z. Cieplak, A. Abal'oshev, M. Berkowski \\ Institute of Physics, Polish Academy of Sciences \\ al. Lotników 32/46, 02-668 Warsaw, Poland
}

M. KOŃCZYKOWSKI

Laboratoire des Solides Irradies, CEA/DSM/DRECAM and CNRS-UMR 7642

École Polytechnique, 91128 Palaiseau Cedex, France

X.M. Cheng, L.Y. Zhu and C.L. Chien

Department of Physics and Astronomy, The Johns Hopkins University

Baltimore, Md 21218, USA

\begin{abstract}
The behavior of vortex pinning induced by the magnetic domain reversal is studied in the ferromagnet/superconductor bilayers in which superconducting film is niobium and the ferromagnetic layers are the $\mathrm{Co} / \mathrm{Pt}$ superlattices with perpendicular magnetic anisotropy. The local magnetic field across narrow ferromagnet/superconductor bilayer stripe is measured using a line of miniature Hall sensors. The pinning is studied for samples with different amount of repeats of $\mathrm{Co} / \mathrm{Pt}$ superlattice, and as a function of temperature.
\end{abstract}

PACS numbers: 74.25.Ha, 74.25.Qt, 74.78.Db, 74.78.Fk

\section{Introduction}

The ferromagnet/superconductor bilayer (FSB) structures are potentially useful devices in which the critical current density of the superconducting (S) layer may be adjusted by the magnetic domain pattern in the ferromagnetic $(\mathrm{F})$ layer. The idea of magnetic pinning in the FSB structures, proposed first by 
Bulaevskii et al. [1], predicts that the magnetic centers (domains) in the $\mathrm{F}$ layer pin the vortex in the $\mathrm{S}$ layer by the magnetic interaction. The proximity effect is eliminated by an insulating buffer layer between the $\mathrm{S}$ and $\mathrm{F}$ layers. The interaction of the magnetic dipole and the vortex introduces the contribution to the energy of the dipole-vortex system, $E=-\boldsymbol{m} \boldsymbol{B}$, where $\boldsymbol{m}$ is the dipole moment, and $\boldsymbol{B}$ is magnetic field of a vortex [2]. It follows that the pinning is sensitive to the relative orientation between $\boldsymbol{m}$ and the external magnetic field $\boldsymbol{H}$. The sensitivity of the pinning in the FSBs to the $\boldsymbol{H}$ direction has been demonstrated in several recent experiments [3-6]. The details of the behavior of the magnetic domain-induced pinning are not yet studied. In particular, it is not known if the pinning depends on the type of the magnetic reversal process (MRP) in the F layer. Depending on the amount of repeats of the $\mathrm{Co} / \mathrm{Pt}$ superlattice, the MRP may proceed by domain wall motion, or by the nucleation of many inverted centers [7]. So far, we have studied the magnetic pinning induced by the first type of MRP, observed in the $\mathrm{Co} / \mathrm{Pt}$ superlattice with small amount of repeats [4-6]. In the present study we compare it with the second type of the MRP. Another issue is the temperature dependence, which we have not addressed previously. It is predicted that the magnetic pinning force is temperature independent when $\lambda<d$, where $\lambda$ is a penetration depth and $d$ is the width of magnetic domains [1].

\section{Samples}

Two different types of FSBs, labeled Nb5 and Nb8, were grown on $\operatorname{Si}(100)$ substrates using high vacuum sputtering system at room temperature. The buffer layers of $10 \mathrm{~nm} \mathrm{Si} \mathrm{were} \mathrm{grown} \mathrm{first,} \mathrm{followed} \mathrm{by} \mathrm{the} \mathrm{superlattice} \mathrm{of} \mathrm{general} \mathrm{formula}$ $[\mathrm{Co}(0.4 \mathrm{~nm}) / \mathrm{Pt}(1 \mathrm{~nm})]_{j}$, where $j=5$ or 8 is the amount of repeats of $\mathrm{Co} / \mathrm{Pt}$ in $\mathrm{Nb} 5$ and Nb8 samples, respectively. Next, $10 \mathrm{~nm}$ thick Si buffer layer and $78 \mathrm{~nm}$ thick $\mathrm{Nb}$ layer was deposited, covered by $3 \mathrm{~nm}$ Si for protection. The superconducting transition temperature, $T_{\mathrm{c}}$, of both $\mathrm{Nb}$ layers was found to be close to $8.8 \mathrm{~K}$. The penetration depth and the coherence length, estimated from the $H_{\mathrm{c} 1}$ and $H_{\mathrm{c} 2}$ measurements were $\lambda(0)=95 \mathrm{~nm}$ and $\xi(0)=35 \mathrm{~nm}$, respectively.

\section{Magnetic reversal process}

Figure 1 shows the hysteresis loop for sample $\mathrm{Nb} 8$, measured in the normal state, at $T=10 \mathrm{~K}$, using two methods: the dc SQUID magnetometer and the anomalous Hall effect (AHE). The loop is typical of samples with perpendicular magnetic anisotropy, with rapid decrease in magnetic moment in the first stage of the MRP, followed by a slower decrease (tail) in the second stage when the remaining uninverted domains are gradually removed by increasing magnetic field. The loop for Nb5 sample is similar, as described previously [4-6], except for coercive fields which are 610 Oe and 700 Oe for the samples Nb5 and Nb8, respectively. The magnetic force microscope (MFM) imaging in the remnant state at $300 \mathrm{~K}$ shows that the MRP in Nb8 sample starts from several nucleation centers, from 

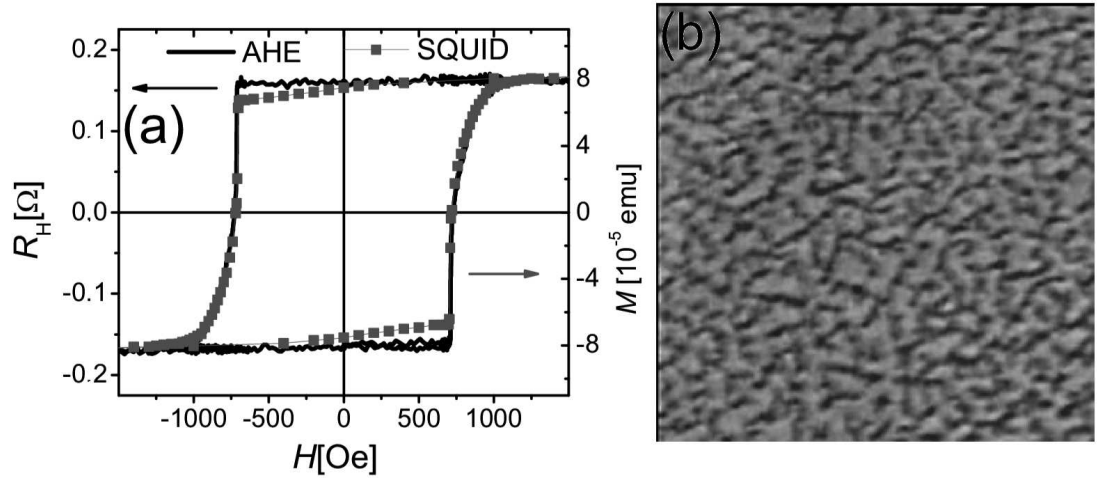

Fig. 1. (a) The hysteresis loops obtained for the sample Nb8 by SQUID (squares) and AHE (line) measurements at $T=10 \mathrm{~K} ; H_{\mathrm{c}}=700 \mathrm{Oe}$; (b) MFM image taken at room temperature for $s_{+}=0.2$. The image size is $40 \times 40 \mu \mathrm{m}^{2}$.

which a fractal structure of inverted domains gradually grows to cover the whole sample. The density of uninverted domains increases and becomes the largest at the beginning of the tail (the image in Fig. 1b is taken at this stage), with the domain width $d$ equal to about $500 \mathrm{~nm}$. On the other hand, we have shown previously that the MRP in the Nb5 sample proceeds by the domain wall motion which leaves behind the maze of uninverted domains [4].

\section{Magnetic pinning}

The magnetic pinning is studied across samples cut into $200 \mu \mathrm{m}$ wide stripes, using the line of 10 miniature Hall sensors, of the area $5 \times 5 \mu \mathrm{m}^{2}$ each, situated $20 \mu \mathrm{m}$ apart. One additional sensor is placed a few millimeters away from the line to measure the background signal. Prior to the measurements, at $T>T_{\mathrm{c}}$, a large magnetic field $H$ is applied to saturate the magnetization of the $\mathrm{F}$ layer. Next, $H$ is reversed and increased up to the magnitude $H_{\mathrm{ExP}}$ to magnetize the F layer. Finally, $H$ is turned off, magnet is quenched, sample is cooled below $T_{\mathrm{c}}$ (to $6.5 \mathrm{~K}$, $7 \mathrm{~K}$ or $7.5 \mathrm{~K}$ ), and the measurements of the local magnetic field are performed in the superconducting state by cycling $\mathrm{H}$ between 90 Oe and -90 Oe. These fields are much lower than the coercive fields, therefore the $\mathrm{F}$ layer remains in the stable remnant state. To describe the MRP we define parameter $s$ which is equal to 1 (0) when all the magnetic moments of the F layer are up (down), and the magnetization at saturation is $M_{\mathrm{s}}\left(-M_{\mathrm{s}}\right)$. At any other state with magnetization $M, s_{ \pm}=\frac{1}{2}\left(M / M_{\mathrm{S}}+1\right)$, where the subscript $+(-)$ indicates the MRP starting from $s=1(s=0)$.

Figure 2a shows the collection of the hysteresis loops measured in the center of the Nb8 sample at $T=7 \mathrm{~K}$ for various values of $s_{+}$. When the $\mathrm{F}$ layer is uniformly magnetized ( $s$ close to 1 or to 0 ), the loops are smooth, narrow and show the shape characteristic of the superconducting film, with positive and negative 

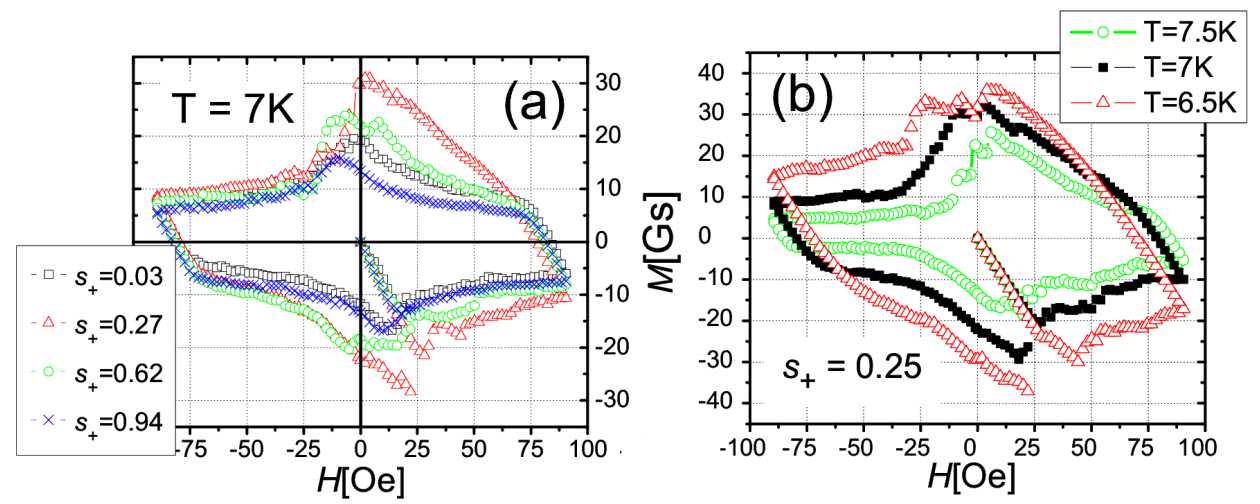

Fig. 2. Hysteresis loops (a) measured at $T=7 \mathrm{~K}$ for different $s$-parameters, (b) measured at $T=6.5 \mathrm{~K}, T=7 \mathrm{~K}, T=7.5 \mathrm{~K}$ for $s_{+}=0.25$.

branches of the hysteresis coupled by the antisymmetric relation. The deviation of $s$ from 1 or 0 leads to a strong asymmetry of the loops with respect to the direction of $H$, indicating the enhancement of vortex pinning when $H$ is positive. This is a result of the magnetic pinning induced by the presence of positive residual magnetic domains, similar to the one described previously in Nb5 sample [4-6]. The magnitude of the pinning enhancement is larger in Nb8 sample by a factor of about 1.5, which may be attributed to a larger magnetic moment resulting from the larger amount of $\mathrm{Co} / \mathrm{Pt}$ repeats. On the other hand, we find that the maximum of the pinning enhancement occurs in both samples for the same $s$ values, i.e. for $s_{+} \approx 0.25$ and $s_{-} \approx 0.75$. In Nb8 sample this corresponds to the maximum density of the uninverted domains. The change of the domain density was not so obvious in Nb5 sample, so it has not been noted previously. The present observation may suggest that in the sample Nb5, with small amount of $\mathrm{Co} / \mathrm{Pt}$ repeats, the maximum of the domain density occurs as well, despite the fact that the MRP proceeds by a domain wall motion.

When $s$ differs from 1 or 0 , in addition to the increased width, the hysteresis loops are broken with sudden tilts, indicative of flux jumps. This behavior is not observed in the case of uniformly magnetized $\mathrm{F}$ layer, so that it is clearly related to the presence of magnetic domains. We suspect that this indicates nonuniform flux flow into the samples.

Figure $2 \mathrm{~b}$ shows the hysteresis loops measured at the center of Nb8 sample for $s_{+}=0.25$ at three different temperatures. The loops broaden significantly with the decreasing $T$. The increasing width is related to the increase in the critical fields and intrinsic critical currents with the decrease in $T$, because the intrinsic pinning centers become more effective at low $T$. Let us note that the asymmetry induced by the magnetic pinning becomes less pronounced at low $T$. This may be explained if we assume that the magnetic pinning is $T$-independent so that at low $T$ it becomes ineffective in comparison with intrinsic pinning. 

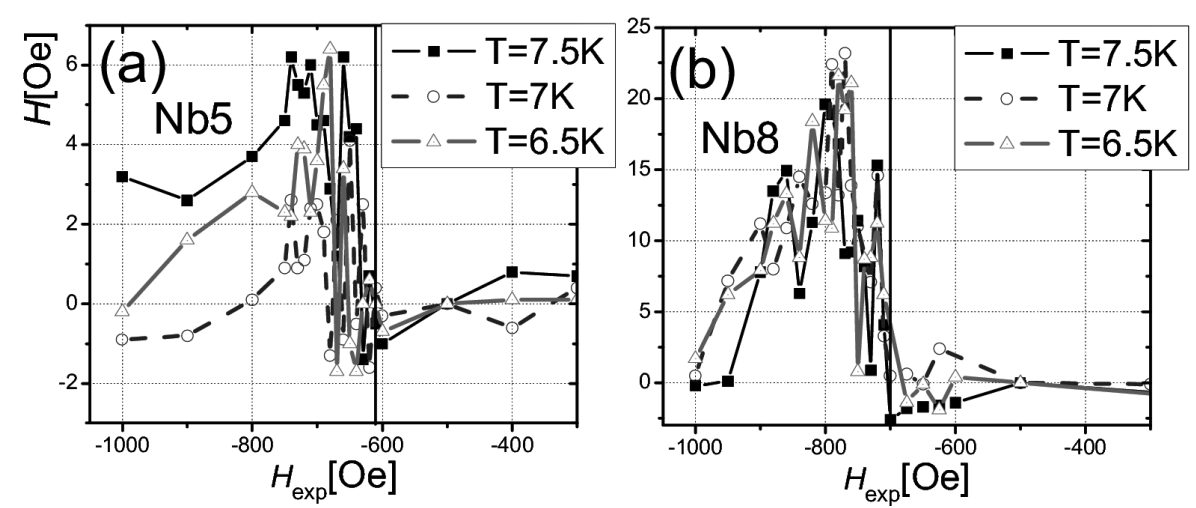

Fig. 3. The field $H$, corresponding to the maximum of the hysteresis loop, versus $H_{\mathrm{ExP}}$. The vertical line indicates the coercive field $H_{\mathrm{c}}$. (a) Nb5 sample, $H_{\mathrm{c}}=610$ Oe; (b) Nb8 sample, $H_{\mathrm{c}}=700$ Oe.

To evaluate the $T$-dependence of the magnetic pinning, we calculate the values of $H$ corresponding to the maxima of the hysteresis loops for different $H_{\mathrm{EXP}}$ (i.e. for different $s$ ). As seen in Fig. 2 a the $H$ is small and negative for $s_{+} \approx 1$, and becomes positive as $s$ approaches $s_{+}=0.25$, so that it is some measure of magnetic pinning. In Fig. 3 we plot the relative value of $H$, calculated with respect to $H$ at $s=1$. We see the sudden appearance of the magnetic pinning for $H_{\mathrm{EXP}}$ just below the coercive field. There is considerable scatter of the data resulting from the flux jumps. Nevertheless, it is clearly seen that the $H$ values are larger in Nb8 sample, which confirms the stronger magnetic pinning in this sample. It is also seen that the values of $H$ measured at different $T$ do not differ significantly suggesting that the pinning force is temperature independent.

\section{Conclusions}

The FSBs with different $F$ layers (different coercive fields) show the enhancement of a vortex pinning during the reorientation of magnetic moments in the $\mathrm{F}$ layer. The enhancement is stronger in samples with larger magnetic moments, but the maximum of magnetic pinning occurs at similar stage of the magnetic reversal process. The pinning force is temperature independent. There is strong influence of flux jumps on the shape of the hysteresis loop.

\section{Acknowledgments}

This work was supported by the French-Polish Bilateral Program "Polonium", grant 2 P03B 04423 of the State Committee for Scientific Research (Poland) and the NSF grant No. DMR00-80031. 


\section{References}

[1] L.N. Bulaevskii, E.M. Chudnovsky, M.P. Maley, Appl. Phys. Lett. 76, 2593 (2000).

[2] M.W. Milosevic, S.V. Yampolskii, F.M. Peters, Phys. Rev. B 66, 174519 (2002).

[3] M. Lange, M.J. Van Bael, V.V. Moshchalkov, Y. Bruynseraede, Appl. Phys. Lett. $\mathbf{8 1}, 322(2002)$.

[4] M.Z. Cieplak, X.M. Cheng, C.L. Chien, H. Sang, J. Appl. Phys. 97, 026105 (2005)

[5] M.Z. Cieplak, Z. Adamus, M. Konczykowski, X.M. Cheng, A. Byczuk, A. Abal'oshev, H. Sang, C.L. Chien, Acta Phys. Pol. A 106, 693 (2004).

[6] M.Z. Cieplak, Z. Adamus, A. Abal'oshev, I. Abal'osheva, M. Berkowski, X.M. Cheng, H. Sang, C.L. Chien, Phys. Status Solidi C 2, 1650 (2005).

[7] J. Pommier, P. Meyer, G. Penissard, J. Ferre, P. Bruno, D. Renard, Phys. Rev. Lett. 65, 2054 (1990) 